\title{
Possible flakes of molecular hydrogen in the early Universe
}

\author{
D. Pfenniger ${ }^{1}$ and D. Puy ${ }^{1,2}$ \\ ${ }^{1}$ Observatory of Geneva, University of Geneva, 1290 Sauverny, Switzerland \\ e-mail: Daniel.Pfenniger@obs .unige.ch \\ ${ }^{2}$ Institute of Theoretical Physics, University of Zürich, Winterthurerstrasse 190, 8057 Zürich, Switzerland \\ e-mail: puy@physik.unizh.ch
}

Received 27 September 2002 / Accepted 3 November 2002

\begin{abstract}
The thermochemistry of $\mathrm{H}_{2}$ and $\mathrm{HD}$ in non-collapsed, non-reionized primordial gas up to the end of the dark age is investigated with recent radiation-matter and chemical reaction rates taking into account the efficient coolant HD, and the possibility of a gas-solid phase transition of $\mathrm{H}_{2}$. In the standard big-bang model we find that these molecules can freeze out and lead to the growth of flakes of solid molecular hydrogen at redshifts $z \approx 6-12$ in the unperturbed medium and under-dense regions. While this freezing caused by the mere adiabatic cooling of the expanding matter is less likely to occur in collapsed regions due to their higher than radiation background temperature, on the other hand the super-adiabatic expansion in voids strongly favors it. Later reionization (at $z \approx 5-6$ ) eventually destroys all these $\mathrm{H}_{2}$ flakes. The possible occurrence of $\mathrm{H}_{2}$ flakes is important for the degree of coupling between matter and radiation, as well as for the existence of a gas-grain chemistry at the end of the dark age.
\end{abstract}

Key words. cosmology: early Universe - cosmology: theory

\section{Introduction}

The period of time between the last scattering of the background radiation photons and the formation of the first bound objects (i.e., the "dark age") still presents interesting unknowns even about the main processes that happened then, as described below.

The studies of the physical and chemical conditions in the post-recombination Universe clearly established that trace amounts of molecules such as $\mathrm{H}_{2}, \mathrm{HD}$ and $\mathrm{LiH}$ were present in the primordial gas (Lepp \& Shull 1984; Dalgarno \& Lepp 1987; Latter \& Black 1991; Puy et al. 1993; Stancil et al. 1996, 1998; Galli \& Palla 1998). A recent and comprehensive review of primordial chemistry is given by Lepp et al. (2002).

Because of its rotational levels, $\mathrm{H}_{2}$ is an important coolant of interstellar gas from $10^{4}$ to $10^{2} \mathrm{~K}$. Despite its lower abundance, the molecule HD is also important, since its allowed dipole rotational transitions provide strong channels coupling radiation and matter at temperatures below $10^{2} \mathrm{~K}$, well below that of the lowest $\mathrm{H}_{2}$ quadrupole transitions (at $512 \mathrm{~K}$ ). Thus primordial molecules can play an important role in the postrecombination Universe, already in the uniform, non-collapsed fraction of the Universe.

Molecules can play an even more crucial role in the first collapsing structures appearing at temperatures typically below a few hundred $\mathrm{K}$, since they offer the possibility to efficiently cool gaseous proto-clouds (see Lahav 1986;

Send offprint requests to: D. Pfenniger,

e-mail: Daniel.Pfenniger@obs.unige.ch
Puy \& Signore 1996, 1997; Uehara et al. 1996, 2000; Abel et al. 1997, 2000). However a definitive understanding of the collapse of the first structures is still lacking, because the coupling of gravity, chemistry, and radiation constitutes a formidable non-linear system for which not all equations are presently well known. Since the system of equations is "stiff", i.e., exponentially sensitive to perturbations, changes in the conclusions can eventually arise if a single factor is modified. For instance Combes \& Pfenniger (1998) show that the fraction of molecular hydrogen can reach unity if the collapsing structures grow not as smooth isolated spheres, but similarly to the fractal structures observed in the galactic ISM.

Zwicky (1959), Takeda et al. (1969), Hirasawa et al. (1969) and Matsuda et al. (1969) pointed out early that molecules could appear during the post-recombination period. The mechanism by which molecules form in the postneutralized Universe is traditionally assumed to be different from the chemistry of the interstellar medium, because the primordial chemistry is supposed to remain a pure gas-phase chemistry.

The first natural extension of molecular physics toward larger matter aggregates is the possibility of a gas-solid phase transition by molecular hydrogen in the primordial gas ${ }^{1}$. This is a central question, because the occurrence of $\mathrm{H}_{2}$ ice grains, or more likely fluffy structures such as flakes, may radically

\footnotetext{
${ }^{1}$ No corresponding ${ }^{4} \mathrm{He}$ gas-solid transition exists. Below $5.2 \mathrm{~K}$, the critical point of ${ }^{4} \mathrm{He}$, down to $0 \mathrm{~K}$ the ${ }^{4} \mathrm{He}$ gas-liquid phase transition occurs at much higher pressure than for the $\mathrm{H}_{2}$ gas-solid transition.
} 
change the chemistry via grain surface reactions, as well as the matter-radiation coupling via photon-grain interactions.

Solid molecular hydrogen ${ }^{2}$, the simplest molecular crystal, is rather special among the molecular crystals because at low pressure the weakly anisotropic $\mathrm{H}_{2}$ molecules are free to rotate and vibrate in the lattice. Further, as for helium, due to its large (comparable to the binding energy) zero-energy motion, quantum effects are important; such crystals are known as quantum crystals. Solid hydrogen has been studied in the laboratory for at least 70 years, and used in several cryogenic and industrial applications, so many of its properties are today well established. A comprehensive review of solid molecular hydrogen is given by Silvera (1980).

In this paper only first order effects associated with the condensed phase of molecular hydrogen are considered. Much more work will be required to describe other possible effects occurring in the non-linear regime following gravitational instability. As it turns out, much depends on the temperature of the matter, and whether matter can be cooled in the interval $\sim 1-2 \mathrm{~K}$ by mere adiabatic expansion.

Mechanical cooling is usually discarded because the overwhelming number of photons in the cosmic background radiation is thought to couple well enough matter and radiation with the few residual electrons by Thomson scattering (see, e.g., the clear discussion by Longair 1995, p. 428). Here we calculate in detail the thermo-chemical evolution with the most recent published rates when the matter temperature cools as $\sim R^{-2}$, faster than the radiation temperature $\sim R^{-1}$ due to the universal expansion ( $R$ is the Universe scale factor). The adiabatic cooling $\sim R^{-2}$ is found to be effective below redshifts $\sim 300$, leaving the possibility to cool non-collapsed, non-reionized matter to very cold temperatures, until most of intergalactic matter is eventually reionized. Current models set the almost complete reionization around redshifts $z \sim 5-6$ (Razoumov et al. 2002).

The outline of this paper is as follows. In Sect. 2, the method of our calculations and the chemistry is described. In Sect. 3, results about the revised thermochemistry are presented, including the calculation of the phase transition to solid $\mathrm{H}_{2}$. The implications are discussed briefly in Sect. 4. Finally, our work is summarized in Sect. 5.

\section{Model}

In most interstellar environments, association catalyzed on grain surfaces is thought to dominate the formation of $\mathrm{H}_{2}$. Thus, molecular hydrogen is generally assumed to form by hydrogen atom recombination on cold grains in dense molecular clouds (Hollenbach \& Salpeter 1970, 1971). At the epoch of recombination where a total absence of dust grains appears justified, surface reactions are ignored. The two standard and principal gas-phase processes are characterized by 1) the $\mathrm{H}_{2}^{+}$ process:

$\mathrm{H}+\mathrm{H}^{+} \longrightarrow \mathrm{H}_{2}^{+}+h v$,

$\mathrm{H}_{2}^{+}+\mathrm{H} \longrightarrow \mathrm{H}^{+}+\mathrm{H}_{2}$,

\footnotetext{
${ }^{2}$ Not to be confused with metallic hydrogen, a high pressure form of hydrogen, found for example inside Jupiter.
}

and 2) the $\mathrm{H}^{-}$process:

$\mathrm{H}+\mathrm{e}^{-} \longrightarrow \mathrm{H}^{-}+h v$,

$\mathrm{H}^{-}+\mathrm{H} \longrightarrow \mathrm{e}^{-}+\mathrm{H}_{2}$.

However, these reactions are coupled with other reactions because the main chemical species available from primordial nucleosynthesis are $\mathrm{H}, \mathrm{H}^{+}, \mathrm{D}, \mathrm{D}^{+}$, He and Li. In particular this simple chemical network must be coupled with the reactions which lead to the formation of HD molecules, because HD is an efficient coolant below $\sim 100 \mathrm{~K}$.

Recently Galli \& Palla (2002) gave an updated review of the deuterium chemistry of the post-recombination Universe from their exhaustive work on the primordial chemistry (Galli \& Palla 1998). The formation of HD in the primordial gas follows the two routes:

$\mathrm{D}^{+}+\mathrm{H}_{2} \longrightarrow \mathrm{H}^{+}+\mathrm{HD}$,

$\mathrm{HD}^{+}+\mathrm{H} \longrightarrow \mathrm{H}^{+}+\mathrm{HD}$.

The radiative association

$\mathrm{H}+\mathrm{D} \longrightarrow \mathrm{HD}+h v$

is very slow in our context.

The chemical reaction rates depend on the temperature of the species or of radiation (for the photoprocesses), and of the density. Thus the chemical kinematics must be coupled with the hydrodynamic equation in the framework of a cosmological model (here the Friedmann model).

A rigorous approach would be to consider four flu$i d s$ : neutrals, positive ions, electrons and photons with their corresponding temperatures in the hydrodynamic equations. However Flower \& Pineau des Forêts (2000) showed a very small difference between the temperature profiles of the neutrals, ions and electrons in the primordial gas. In fact, the coupling through Coulomb scattering between the electrons and the ions and the neutrals is sufficiently strong at the postrecombination epoch. In the following $T_{\mathrm{m}}$, is the common temperature of the neutrals, ions, and electrons, which we call matter temperature.

The temperature of the cosmic background radiation (CBR) is given by:

$\frac{\mathrm{d} T_{\mathrm{r}}}{\mathrm{d} t}=-H(z) T_{\mathrm{r}}$,

a relation which has been observationally confirmed by Srianand et al. (2000), at the redshift $z=2.138$, with absorption lines from the first and second fine-structure levels of neutral carbon atoms in an isolated cloud of gas.

$H(z)$ is the Hubble parameter at the redshift $z$, defined from the scale factor $R$, and $H_{0}$ (the Hubble constant) is its present value:

$$
\begin{aligned}
H(z) & =\dot{R} / R \\
& =H_{0}\left[\Omega_{\mathrm{r}, 0}(1+z)^{4}+\Omega_{\mathrm{m}, 0}(1+z)^{3}+\Omega_{K, 0}(1+z)^{2}+\Omega_{\Lambda, 0}\right]^{\frac{1}{2}},
\end{aligned}
$$

where the $\Omega_{, 0}$ are constants with the present values, constrained by the Friedman-Lemaître condition:

$\Omega_{\mathrm{r}, 0}+\Omega_{\mathrm{m}, 0}+\Omega_{K, 0}+\Omega_{\Lambda, 0}=1$. 
$\Omega_{\mathrm{r}, 0}$, the radiation density parameter, is given by:

$\Omega_{\mathrm{r}, 0}=\frac{a T_{3 \mathrm{~K}}^{4}}{c^{2}} \frac{8 \pi G}{3 H_{\mathrm{o}}^{2}}\left(1+f_{v}\right)$ with $f_{v}=\frac{21}{8} \times\left(\frac{4}{11}\right)^{4 / 3}$,

where $a=4 \sigma / c$ is the radiation constant related to the StefanBoltzmann constant $\sigma, T_{3 \mathrm{~K}}=2.726 \mathrm{~K}$ is the present radiation temperature (Mather et al. 1994). The neutrino contribution to the radiation density for three massless neutrino types is noted $f_{v}, G$ is the gravitational constant, and $c$ is the speed of light. $\Omega_{\mathrm{m}, 0}$ is the matter density parameter (i.e., baryons and other non-baryonic matter), $\Omega_{K, 0}$ is the curvature parameter, and $\Omega_{\Lambda, 0}$ is the mass-energy density parameter associated with the Einstein's cosmological constant $\Lambda$, or with quintessence (Wetterich 1988; Peebles \& Ratra 2002). In our context, $\Omega_{\Lambda, 0}$ will be held constant, fixed for $z=0$.

The evolution of the gas temperature $T_{\mathrm{m}}$ is governed by the equation (see Puy et al. 1993):

$\frac{\mathrm{d} T_{\mathrm{m}}}{\mathrm{d} t}=-2 H(z) T_{\mathrm{m}}+\Psi_{\text {Compton }}+\Psi_{\mathrm{mol}}$.

$\Psi_{\text {Compton }}$ characterizes the energy transfer from radiation to matter via Compton scattering of CBR photons on electrons (Peebles 1968):

$\Psi_{\text {Compton }}=\frac{8}{3} \frac{\sigma_{T} a}{m_{\mathrm{e}} c} T_{\mathrm{r}}^{4}\left(T_{\mathrm{r}}-T_{\mathrm{m}}\right) x_{\mathrm{e}}$.

$\sigma_{T}$ defines the Thomson cross-section and $m_{\mathrm{e}}$ the electron mass. $x_{\mathrm{e}}$ is the electron abundance (i.e., the ionization fraction). $\Psi_{\text {mol }}$ is the energy transfer via excitation and de-excitation of molecular transition. However, in the post-recombination context this thermal function is a heating function and gives a slight contribution to the evolution of matter temperature (see Puy et al. 1993).

This series of differential equations is completed by the redshift equation:

$\frac{\mathrm{d} z}{\mathrm{~d} t}=-H(z)(1+z)$

and by the equation of the numerical density of baryons $n$ :

$\frac{\mathrm{d} n}{\mathrm{~d} t}=-3 H(z) n$,

which just describes the dilution of the conserved number of baryons in the expanding volume.

Each chemical species $n_{\mathrm{s}}$ has a similar behavior, completed with the chemical network:

$\frac{\mathrm{d} n_{\mathrm{s}}}{\mathrm{d} t}=-3 H(z) n_{\mathrm{s}}+\left(\frac{\mathrm{d} n_{\mathrm{s}}}{\mathrm{d} t}\right)_{\text {chem }}$,

where the chemical network $\left(\frac{\mathrm{d} n_{\mathrm{s}}}{\mathrm{d} t}\right)_{\text {chem }}$ has the form:

$\left(\frac{\mathrm{d} n_{\mathrm{s}}}{\mathrm{d} t}\right)_{\mathrm{chem}}=\sum_{i, j} \alpha_{i j} n_{i} n_{j}-\beta_{i j} n_{\mathrm{s}}$

The first terms of the sum define the $s$ formation process $(i+j \rightarrow s)$ associated with the reaction rate $\alpha_{i j}$, the second term characterizes the destruction process $(s \rightarrow i+j)$ with the reaction rate $\beta_{i j}$. Some reactions rates $\alpha_{i j}$ and $\beta_{i j}$ are given in the Appendix.
Table 1. Standard cosmological model (S-model): parameters and initial relative abundances at $z=10^{4}$.

\begin{tabular}{|l|c||l|c|}
\hline \hline$H_{0}$ & $67 \mathrm{~km} \mathrm{~s}^{-1} \mathrm{Mpc}^{-1}$ & $Y_{\mathrm{p}}$ & 0.24 \\
$\Omega_{\mathrm{r}, 0}$ & $9.265 \times 10^{-5}$ & {$[\mathrm{D}]$} & $3.3 \times 10^{-5}$ \\
$\Omega_{\mathrm{m}, 0}$ & $\sim 0.29990$ & {$[\mathrm{Li}]$} & $2.1 \times 10^{-10}$ \\
$\Omega_{\mathrm{b}, 0}$ & 0.0535 & {$\left[\mathrm{H}_{2}\right]$} & $10^{-40}$ \\
$\Omega_{K, 0}$ & 0 (flat Universe) & {$[\mathrm{HD}]$} & $10^{-50}$ \\
$\Omega_{\Lambda, 0}$ & 0.7 & {$[\mathrm{LiH}]$} & $10^{-50}$ \\
\hline
\end{tabular}

\subsection{Initial conditions}

With stiff systems of equations, it is obviously important to use the most accurate available physical constants and parameters. In the context of the primordial chemistry the knowledge of the abundances of the primordial nuclei is crucial. From the latest determination of primordial helium (i.e., $Y_{\mathrm{p}} \sim 0.24$ ) and deuterium $\left(\mathrm{D} / \mathrm{H} \sim 3.3 \times 10^{-5}\right)$ abundances given by Tytler et al. (2000) we can deduce the baryon density, which is in good agreement with the cosmic microwave background measurements of Boomerang and Maxima (de Bernardis et al. 2000; Jaffe et al. 2001): $\Omega_{\mathrm{b}, 0} h^{2} \sim 0.024$. These experiments find a peak in the angular power spectrum of the microwave background, consistent with that expected for cold dark matter models in a flat Universe (i.e., $\Omega_{K, 0}=0$ ).

The observations of type Ia supernovae (Perlmutter et al. 1999; Riess et al. 1998) suggest that the Universe may be presently dominated by an additional dark energy. Combined observations of type Ia supernovae, cosmic microwave background anisotropy (Jaffe et al. 2001) and cluster evolution (Bahcall \& Fan 1998) for which the results have been done in the form of likelihood contours in the $\Omega_{\mathrm{m}, 0}$ and $\Omega_{\Lambda, 0}$ plane, yield

$\Omega_{\mathrm{m}, 0} \sim 0.3$ and $\Omega_{\Lambda, 0} \sim 0.7$.

In our case $\Omega_{\mathrm{m}, 0} \sim 0.2999$ and $\Omega_{\mathrm{r}, 0} \sim 9.265 \times 10^{-5}$, in order to verify the Friedman-Lemaître condition (Eq. (7)). We adopt the Hubble constant $H_{0}=67 \mathrm{~km} \mathrm{~s}^{-1} \mathrm{Mpc}^{-1}$ given by Freedman (2000). We summarize the parameters of our standard model (S-model) in Table 1.

In other models (see discussion) we will vary only the value of the baryonic fraction $\Omega_{\mathrm{b}, 0}$. Cosmochemistry evolution is not sensitive to the $\Omega_{\Lambda, 0}$ parameter. Molecular formation appears in the redshift range $(100,1000)$ whereas $\Omega_{\Lambda, 0}$ plays a role in the Hubble parameter, see Eq. (6), at redshift below $z \sim 5$ (see Carroll et al. 1992). We will fix this last parameter to the value $\Omega_{\Lambda, 0}=0.7$.

\subsection{Chemical evolution}

The numerical integration of the coupled chemical and hydrodynamical equations is an initial value problem for stiff differential equations. We use the chemical network developed by Galli \& Palla (1998, 2002), except the hydrogen and deuterium recombination which is calculated from the reaction rates given by Abel et al. (1997), see Appendix. 

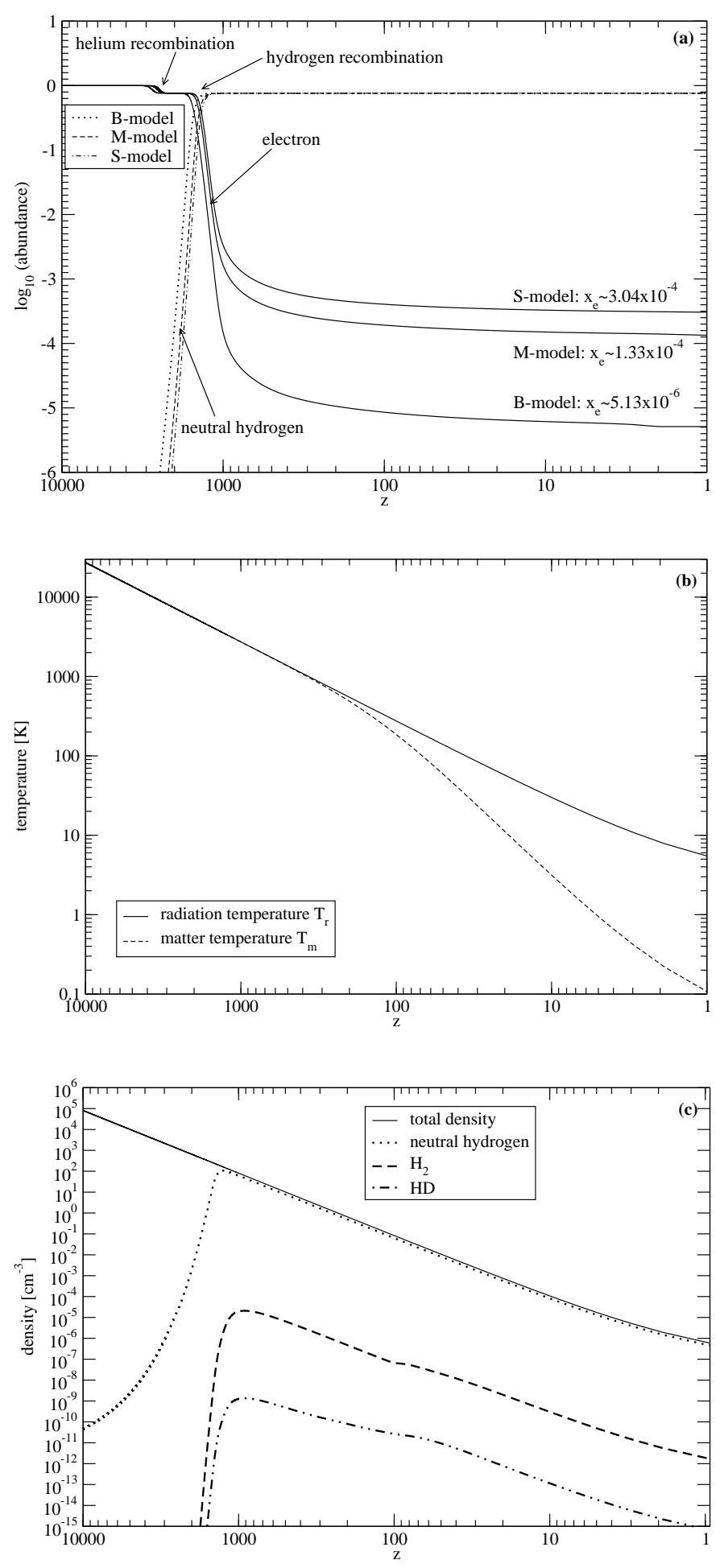

Fig. 1. a) Helium and hydrogen recombination for a flat Universe with $H_{0}=67 \mathrm{~km} \mathrm{~s}^{-1} \mathrm{Mpc}^{-1}$ and $\Omega_{\Lambda, 0}=0.7$. The S-model corresponds to the baryonic fraction $\Omega_{b, 0}=0.0535$, the M-model to $\Omega_{b, 0}=0.1$, and the B-model to $\Omega_{b, 0}=1$. b) Thermal decoupling between radiation and matter in the S-model. c) Comparison of the $\mathrm{H}_{2}$ and HD density in the S-model. After a transient growth $\mathrm{H}_{2}$ and $\mathrm{HD}$ follow the evolution of the neutral density, which means that the fractional abundance becomes almost constant below $z \sim 500$.
We start our integration at redshift $z_{i}=10^{4}$. At this value it is particularly important to take into account ${ }^{3}$ the radiation contribution $\Omega_{\mathrm{r}, 0}$ in order to calculate the age of the Universe $t_{i}$ at redshift $z_{i}$ :

$t_{i}=\frac{1}{H_{0}} \int_{1+z_{i}}^{\infty}\left[\Omega_{\mathrm{r}, 0} x^{4}+\Omega_{\mathrm{m}, 0} x^{3}+\Omega_{K, 0} x^{2}+\Omega_{\Lambda, 0}\right]^{-1 / 2} \frac{\mathrm{d} x}{x}$.

The time $t_{i}$ gives a timescale for the initial integration of the set of Eqs. (5)-(14), which is solved by Gear's method (Gear 1971).

In Fig. 1a we show the helium and hydrogen recombination which give the abundance of the free electrons. The slight decreasing of the electronic fraction $x_{\mathrm{e}}$ at $z \sim 2485$ corresponds to the helium recombination. At the redshift $z \sim 1360$ the medium becomes neutral (hydrogen recombination: neutral hydrogen is dominant). In all of these cosmological models the fraction of electrons, $x_{\mathrm{e}}$, is low at $z=1$. For the S-model $x_{\mathrm{e}}=3.04 \times 10^{-4}$ is in close accordance with the results of Galli \& Palla (1998) where $x_{\mathrm{e}} \sim 3.02 \times 10^{-4}$. Before and around recombination, the thermal history depends on the tight coupling between radiation and matter resulting from Thomson scattering. Well after recombination, the free electrons quasi vanished - i.e. $x_{\mathrm{e}} \sim$ few $\left[10^{-4}-10^{-6}\right]$, see Fig. 1a. The evolution of the mean temperature of radiation and of matter is shown in Fig. 1b. The subsequent evolution of matter temperature is mainly caused by the dilatation of the Universe (first term in the second member of Eq. (9)), despite the very high numerical photon density at low redshifts (see Longair 1995; Partridge 1995). We notice that the thermal decoupling is effective at redshift $z_{\mathrm{dec}} \sim 498$ (we define $z_{\mathrm{dec}}$ the redshift below which the ratio $T_{\mathrm{m}} / T_{\mathrm{r}}$ is lower than 0.99). The non-collapsed matter temperature can drop much below the radiation temperature, unless some other coupling channels not included in our model are effective. The possibility of cooling matter well below radiation is crucial for the possibility to form $\mathrm{H}_{2}$ flakes. In Fig. 1c we have plotted the evolution of the density. After the thermal decoupling the total density is mainly due to neutral hydrogen. The $\mathrm{H}_{2}$ density rises through two processes: the first step corresponds to the $\mathrm{H}_{2}^{+}$ channel and the second one to the $\mathrm{H}^{-}$channel. The HD density rises also in two steps with a transition around $z \sim 100$, the $\left(\mathrm{D}^{+}, \mathrm{H}_{2}\right)$ channel and the $\left(\mathrm{HD}^{+}, \mathrm{H}\right)$ channel.

\section{Thermochemistry}

Fifty years ago, van de Hulst (1949) mentioned the possibility of the existence of solid $\mathrm{H}_{2}$ in the interstellar medium, which implies the possibility of a gas-solid phase transition. This possibility was elaborated by Wickramasinghe \& Reddish (1968) and Hoyle et al. (1968). In the context of baryonic dark matter as cold $\mathrm{H}_{2}$ Pfenniger \& Combes (1994) and Wardle \& Walker (1999) found also that solid $\mathrm{H}_{2}$ can play an important role.

This form of molecular hydrogen was already well known in the laboratory for a long time. For example, Harold Urey in

\footnotetext{
${ }^{3}$ In our cosmological model, the equilibrium redshift (i.e., between matter and radiation density) is $1+z_{\mathrm{eq}}=\Omega_{\mathrm{m}, 0} / \Omega_{\mathrm{r}, 0}=$ $\frac{3 H_{0}^{2}}{8 \pi G} \frac{c^{2}}{a T_{3 \mathrm{~K}}^{4}} \frac{1}{1+f_{V}} \Omega_{\mathrm{m}, 0} \sim 3235$. This value justifies that it is necessary to consider the radiative component.
} 
his Nobel prize lecture (1935) described how this phase transition was used to easily separate the $\mathrm{H}_{2}$ and $\mathrm{D}_{2}$ isotopes, thank to their slightly different triple points.

From cryogenic industry tables (L'Air Liquide 1976), the sublimation curve of para-hydrogen can be well fit with the following equation (Pfenniger \& Combes 1994):

$p_{\text {sat }}=5.7 \times 10^{20} T_{\mathrm{m}}^{5 / 2} \exp \left(-\frac{91.75}{T_{\mathrm{m}}}\right) \mathrm{K} \mathrm{cm}^{-3}$

in the range of matter temperature $T_{\mathrm{m}}$ between $1 \mathrm{~K}$ and the triple point temperature at $T_{3}=13.8 \mathrm{~K}$. According to L'Air Liquide (1976) p. 890, below about $20 \mathrm{~K}$ over $99 \%$ of normal $\mathrm{H}_{2}$ is in the para form. The asymptotic mixture containing the maximum of ortho- $\mathrm{H}_{2}$ takes place between about 20 to $300 \mathrm{~K}$, above which the ortho/para ratio converges toward 3. However, Flower \& Pineau des Forets (2000), investigating the ortho/para $\mathrm{H}_{2}$ ratio, found that this ratio decreases to a frozen value of about 0.25 for $z<20$ due to the low density of the main conversion agent, $\mathrm{H}^{+}$. We will see such a different ortho/para ratio has a very small effect in the result about the $\mathrm{H}_{2}$ condensation redshift.

If we suppose an ideal $\left(\mathrm{H}_{2}-\mathrm{HD}\right)$ gas $^{4}$, the ratio $\xi$ between the $\mathrm{H}_{2}$-gas pressure and the saturation pressure becomes:

$\xi=\frac{p_{\mathrm{H}_{2}}}{p_{\text {sat }}} \approx 2.63 \times 10^{-21} n_{\mathrm{H}_{2}} T_{\mathrm{m}}^{-3 / 2} \exp \left(\frac{91.75}{T_{\mathrm{m}}}\right)$,

where $n_{\mathrm{H}_{2}}$ is the $\mathrm{H}_{2}$ density (in $\mathrm{cm}^{-3}$, see Fig. 1c). Although $\mathrm{HD}$ has a dipole moment when $\mathrm{H}_{2}$ has quadrupolar transitions, the thermodynamics of HD gas is assumed to be very close to $\mathrm{H}_{2}$ (without distinction between $\mathrm{H}_{2}$ and HD).

In Fig. 2a the ratio $\xi$ is plotted for three different cosmological models: 1) S-model: the standard cosmological model, 2) M-model: model with $\Omega_{\mathrm{b}, 0}=0.1,3$ ) B-model: model with $\Omega_{\mathrm{b}, 0}=\Omega_{\mathrm{m}, 0} \sim 0.3$. For all of these models $\left.\Omega_{\Lambda, 0}=0.7\right)$. We define $z_{\text {flake }}$ as the redshift for which below this boundary the ratio $\xi$ is greater than 1 , and thus leads to the possibility to freeze the $\left(\mathrm{H}_{2}-\mathrm{HD}\right)$ gas.

We find that $z_{\text {flake }} \sim 12$ for the B-model, $z_{\text {flake }} \sim 7$ for the M-model, and $z_{\text {flake }} \sim 6$ for the S-model. The redshift $z_{\text {flake }}$ is larger in the B-model because the intrinsic baryon density is more important.

In Fig. 2 we show also the very slight influence of the molecule rotation factor, $5 / 2$ or $3 / 2$, in the $\mathrm{H}_{2}$ pressure. The redshift $z_{\text {flake }}$ is not essentially affected. The same remark is valid when the ortho/para ratio is taken as 0.25 instead of 0 , since $p_{\mathrm{H}_{2}}$ is lowered by only a factor 0.75 . In Fig. $2 \mathrm{~b}$ we compare the pressure $p_{\text {sat }}$ and the partial pressure $p_{\mathrm{H}_{2}}(\mathrm{~S}$-model) below the triple point. Obviously, the insensitivity of $z_{\text {flake }}$ to variations of $p_{\mathrm{H}_{2}}$ results from the steepness of the $\mathrm{H}_{2}$ sublimation curve.

\section{Discussion}

What we have shown above is that below a critical redshift a necessary condition to form $\mathrm{H}_{2}$ solid grains is fulfilled.

${ }^{4}$ In this section, $p_{\mathrm{H}_{2}}=\frac{3}{2} n_{\mathrm{H}_{2}} T_{\mathrm{m}}$ where we neglect the rotation and vibration of the molecule, a valid approximation in the $\mathrm{H}_{2}$ solidification range $0-13.8 \mathrm{~K}$.
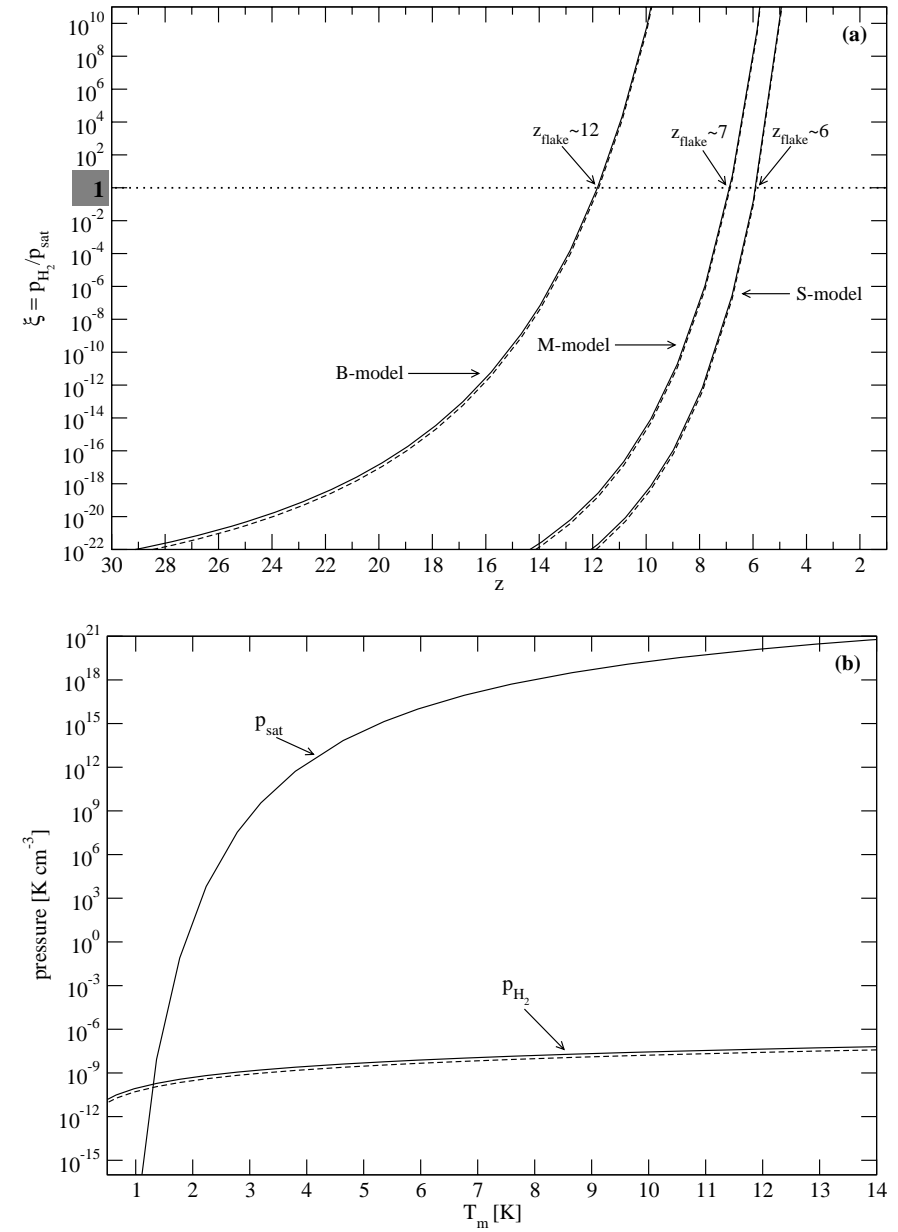

Fig. 2. a) Comparison of the ratio between the gas pressure and the saturation pressure $p_{\text {sat }}$, for $\mathrm{H}_{2}-\mathrm{HD}$ gas between $1 \mathrm{~K}$ and $T_{3}=13.8 \mathrm{~K}$ (i.e., range of redshift between 1 and 30), $z_{\text {flake }}$ is the redshift below which flakes form; B-model: $\Omega_{\mathrm{b}, 0} \sim 1$, M-model: $\Omega_{\mathrm{b}, 0} \sim 0.1$, S-model: $\Omega_{\mathrm{b}, 0} \sim 0.0535$ (for all of these models $\Omega_{\Lambda, 0}=0.7$ ). For each model, the solid curves corresponds to molecules with allowed rotation, while the dashed curves to non-rotating molecules. b) Comparison of the saturation pressure $p_{\text {sat }}$ with the $\mathrm{H}_{2}$ partial pressure $p_{\mathrm{H}_{2}}$, for the $\mathrm{S}$ model as a function of temperature.

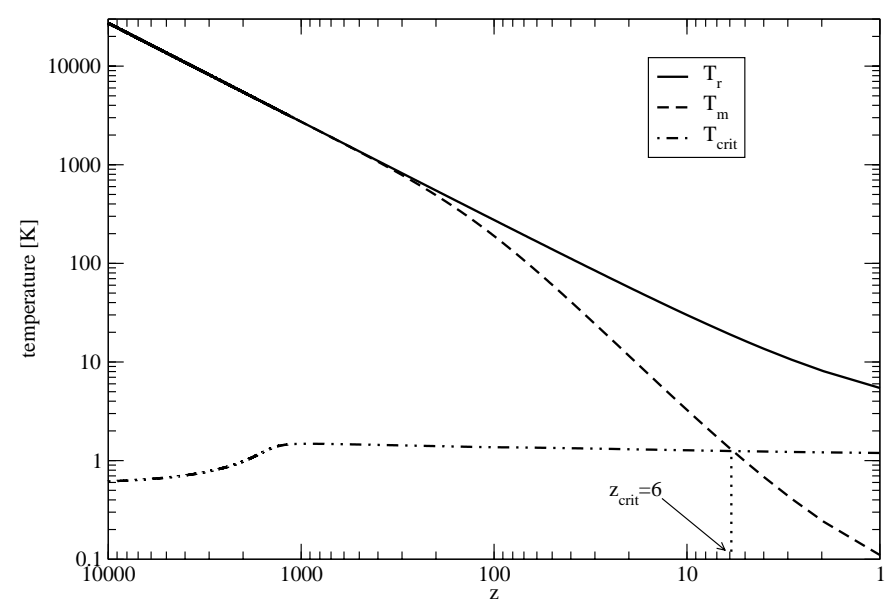

Fig. 3. Evolution of temperatures of matter and radiation. The critical temperature is reached at redshift 6; below this redshift $\mathrm{H}_{2}$ could stick to the surface of ice. 
However, this condition is not sufficient, in reality several complications may prevent or favor the formation of solid $\mathrm{H}_{2}$. We discuss below some of these complications, as well as some consequences in case solid $\mathrm{H}_{2}$ does form.

\subsection{Coupling of chemistry and $\mathrm{H}_{2}$ flakes}

Sandford \& Allamandola (1993) showed experimentally that $\mathrm{H}_{2}$ molecules can stick to the surfaces of usual ices, and argued that gas-phase $\mathrm{H}_{2}$ freezes out onto classical dust grains. Thus $\mathrm{H}_{2}$-containing ices may be fairly common in dense molecular clouds.

On the other hand, solid hydrogen is a quantum solid with a large zero-point energy (Silvera 1980), which means that contrary to usual dust grains, atoms or molecules adsorbed on the surface of $\mathrm{H}_{2}$ ice do not stick at fixed lattice sites but move easily around to go to the lowest free energy configuration. The consequence is that surface chemical reactions may be thus greatly accelerated on $\mathrm{H}_{2}$ ice.

Atoms or molecules stick on the $\mathrm{H}_{2}$ ice surface below a critical temperature which is estimated by Sandford \& Allamandola:

$$
T_{\text {crit }}=\frac{100}{56.46-\ln \left(n_{\mathrm{H}_{2}}\right)} \mathrm{K} .
$$

In Fig. 3, we compare the evolution of the radiation and matter temperatures with the evolution of the critical temperature. We see that the critical redshift $z_{\text {crit }}=6$, i.e., also the critical redshift for which the temperature of $\left(\mathrm{H}_{2}-\mathrm{HD}\right)$ gas is below the critical temperature (Eq. (19)). The value of $z_{\text {crit }}$ is remarkably close to the threshold redshift leading to the formation of $\mathrm{H}_{2}$ flakes (Figs. 2a and 2b), which is not unexpected since condensation and sticking phenomena are both closely related to the inter-molecular force.

Thus, we can imagine that once the first flakes form, the formation of $\mathrm{H}_{2}$ and HD may be enhanced by neutral hydrogen reactions on the flake, which in turn increase the $\mathrm{H}_{2}$ vapor pressure, increasing the flake growth.

Sandford \& Allamandola (1993) speculated on the possibility that pure $\mathrm{H}_{2}$ ices might exist in intergalactic and extragalactic space. This last hypothesis had received some attention previously (Lee et al. 1971 or Hegyi \& Olive 1986).

\subsection{Coupling of background radiation and $\mathrm{H}_{2}$ flakes}

However, in the still neutral and not collapsed regions of the Universe, the background radiation is warmer than the adiabatically expanding matter. If $\mathrm{H}_{2}$ flakes form, a new channel opens for coupling matter and radiation, since solids possess numerous phonon bands able to interact with infrared radiation.

The most prominent absorption bands in solid $\mathrm{H}_{2}$ occurs in the interval 1.89-2.50 $\mu \mathrm{m}$ (e.g., Allin et al. 1955), and at higher frequency in the interval 1.06-1.24 $\mu \mathrm{m}$ (Varghese et al. 1987). They are in part caused by the vibrational and rotational quadrupolar absorption as for the isolated molecule, and by the main vibrational mode between two adjacent molecules in the lattice.
A particularity of solid $\mathrm{H}_{2}$ is that the molecules in the crystal at low pressure are almost free to vibrate and rotate, molecular transitions of low quantum numbers being allowed. Therefore it may be useful to mention a few other transitions for free molecules in the far infrared. Zwicky (1959) mentioned a line at $85 \mu \mathrm{m}$ associated to the $\mathrm{H}_{2}$ ortho-para transition, but this transition is very slow, of order of $300 \mathrm{yr}$. Particularly interesting for the dark age context is the HD dipolar rotational transition around $114 \mu \mathrm{m}$ (e.g., Lee et al. 1988) which, despite a low HD abundance, is relevant since the transition is dipolar, and the background radiation particularly intense in the far infrared.

Thus any coupling of radiation with solid $\mathrm{H}_{2}$ should increase the matter temperature, which acts to sublimate $\mathrm{H}_{2}$ flakes, a self-destructive process. At any redshift an equilibrium temperature should be reached where the condensation and sublimation rates are equal. Several uncertainties prevent us from estimating these time-dependent rates, such as the radiation-flake coupling which depends on the size, geometry and crystalline structure of the $\mathrm{H}_{2}$ flakes, and also the chemistry coupling mentioned above. More "cosmological" laboratory experiments simulating aspects of the physico-chemical conditions during the dark age would be useful.

\subsection{Heating from latent heat}

The formation of $\mathrm{H}_{2}$ ice liberates molecular binding energy in the form of heat, the latent heat. In the range $1-3 \mathrm{~K}$ the latent heat amounts to $92-99 \mathrm{kcal} \mathrm{kg}^{-1}$ (L'Air Liquide 1976), or 2262 times the molecule kinetic energy $\frac{3}{2} k T$. Since the $\mathrm{H}_{2}$ abundance is then of the order of $10^{-6}$, clearly the latent heat can easily be shared with the neutral gas without causing any significant temperature increase.

\subsection{Destruction of $\mathrm{H}_{2}$ flakes by reionization}

At redshifts 5-9, one expects that a growing fraction of the non-collapsed Universe is filled by the UV radiation produced by the first collapsed and light emitting structures, stars and quasars (see, e.g., the models of Razoumov et al. 2002). This means that at redshift 6-7 possibly most of the diffuse gas has already passed through a ionization front, and is reheated to high temperatures. So the formation of flakes may be partly or totally suppressed if the reionization occurs early enough, and propagates in every corner of the Universe. Voids are the last regions to be reionized, for reasons well discussed by Razoumov et al. (2002): in brief, UV radiation percolates through ionization fronts and thus ionizes the dense regions first. The number of UV photons necessary to reach and ionize voids is therefore much larger than, as earlier assumed, for a UV optically thin medium bathed by a general radiation field. The result is to delay the full reionization of the Universe, leaving some more time for $\mathrm{H}_{2}$ freezing, particularly in voids.

\subsection{Formation of $\mathrm{H}_{2}$ flakes in voids}

An additional mechanism enhancing $\mathrm{H}_{2}$ freezing occurs in regions expanding faster than the Universe, i.e., in voids, since there the adiabatic cooling is even stronger. The essential factor favoring $\mathrm{H}_{2}$ freezing is the temperature. If the void matter 
reaches a temperature around $1-2 \mathrm{~K}$ the formation of $\mathrm{H}_{2}$ flakes is very much facilitated because the saturation pressure drops extremely fast, by over 27 dex in this range, as can be derived from Eq. (17), or Fig. 2b. Since in an adiabatic mono-atomic gas $T \propto \rho^{\gamma-1}$, a region that is 10 times underdense with respect to the average would be, for $\gamma=5 / 3,10^{2 / 3}=4.6$ times colder. Since $T_{\mathrm{m}} \propto(1+z)^{2}$, the redshift at which $\mathrm{H}_{2}$ reaches the critical freezing temperature would about double, bringing it at a much darker epoch $z>12$.

\subsection{Formation of $\mathrm{H}_{2}$ flakes in collapsing structures}

On the other hand, if gas radiative cooling $\propto n^{2}$ is sufficiently strong, in collapsing regions eventually the temperature increase by adiabatic compression is canceled by efficient radiative cooling. Primordial molecules such as HD are particularly efficient for cooling at temperatures below $100 \mathrm{~K}$.

The prediction of the temperature runs in gravitational collapsing structures is highly non-trivial since the negative specific heat of self-gravitating systems may lead to opposite behaviours than those in familiar thermal systems. Observations of the galactic ISM show that unstable gas tends to adopt a fractal structure over 4-5 orders of magnitude, and the smallest gas clouds may remain very cold and dense. In this context, Pfenniger \& Combes (1994) argue that cold $\mathrm{H}_{2}$ gas may be a candidate for baryonic dark matter provided that the smallest elements of the gas fractal structure, the clumpuscules, are sufficiently cold and compact. In their postulated conditions set essentially by the virial theorem, at low redshifts $\left(T \approx 3 \mathrm{~K}, n \approx 10^{9} \mathrm{~cm}^{-3}\right.$ ) they noted that the conditions are met to form solid $\mathrm{H}_{2}$ due to a much higher gas pressure than the one contemplated above in the unperturbed Hubble flow. However if the cold collapsed structures reach the CBR temperature from above, clearly condensed $\mathrm{H}_{2}$ (in solid or liquid forms) cannot form below the redshift at which the radiation is at the $\mathrm{H}_{2}$ critical point temperature, at $33.4 \mathrm{~K}$. In other words, the largest redshift for the occurrence of condensed $\mathrm{H}_{2}$ in equilibrium with the CBR in dense but cold clumps is at $z=33.4 / 2.726-1=11.25$.

\section{Conclusions}

In the current understanding of the formation of the first bound structures during the dark age, the possibility that solid hydrogen flakes exist and modify the subsequent evolution must be considered. For regions in which the growth of inhomogeneities can be neglected, in widely different Universe models our chemical calculations show that between redshifts 6 and $12 \mathrm{H}_{2}$ flakes may start to form. The subsequent chemistry and matter-radiation coupling should be significantly altered. Since several factors that can either damp or increase the formation of $\mathrm{H}_{2}$ flakes, clearly one must take into account that the late dark age thermochemistry is likely to be much more complex that earlier admitted.

However at these redshifts most of the first structure formation scenarios predict an already well advanced and widespread stage of reionization, which casts uncertainties about which processes win, or which fraction of the Universe between voids and collapsed regions are concerned by $\mathrm{H}_{2}$ flake formation. The appearance of snowflakes at the end of the dark age would constitute the first "cosmic winter".

Acknowledgements. Part of this work was supported by the TomallaStiftung and the Swiss National Science Foundation. The authors gratefully acknowledge useful discussions with F. Combes, M. Signore, and F. Melchiorri. Part of the preparation of this manuscript has been done during a stay at the Aspen Center for Physics. We are grateful to the referee, Daniele Galli, for constructive comments.

\section{Appendix}

We use the reactions rates $\alpha_{i j}$ and $\beta_{i j}$ and photo-processes given by Galli \& Palla (1998, 2002), except the hydrogen and deuterium recombination:

$\mathrm{H}^{+}+\mathrm{e}^{-} \rightarrow \mathrm{H}+\gamma$

$\mathrm{D}^{+}+\mathrm{e}^{-} \rightarrow \mathrm{D}+\gamma$

with a reaction rate $\alpha_{\text {rec }}$ (in $\mathrm{cm}^{-3} \mathrm{~s}^{-1}$ ), which is calculated from the approximation given by Abel et al. (1997):

$$
\begin{aligned}
\alpha_{\text {rec }}=\exp [ & -28.6130338-0.72411256 \ln \left(T_{\mathrm{m}}\right) \\
& -2.02604473 \times 10^{-2} \ln ^{2}\left(T_{\mathrm{m}}\right) \\
& -2.38086188 \times 10^{-3} \ln ^{3}\left(T_{\mathrm{m}}\right) \\
& -3.21260521 \times 10^{-4} \ln ^{4}\left(T_{\mathrm{m}}\right) \\
& -1.42150291 \times 10^{-5} \ln ^{5}\left(T_{\mathrm{m}}\right) \\
+ & 4.98910892 \times 10^{-6} \ln ^{6}\left(T_{\mathrm{m}}\right) \\
+ & 5.75561414 \times 10^{-7} \ln ^{7}\left(T_{\mathrm{m}}\right) \\
& -1.85676704 \times 10^{-8} \ln ^{8}\left(T_{\mathrm{m}}\right) \\
& \left.-3.07113524 \times 10^{-9} \ln ^{9}\left(T_{\mathrm{m}}\right)\right]
\end{aligned}
$$

where $T_{\mathrm{m}}$ is in $\mathrm{eV}$. We consider also the reverse reaction (i.e., photoionization of neutral hydrogen and neutral deuterium):

$$
\begin{aligned}
& \mathrm{H}+\gamma \rightarrow \mathrm{H}^{+}+\mathrm{e}^{-} \\
& \mathrm{D}+\gamma \rightarrow \mathrm{D}^{+}+\mathrm{e}^{-}
\end{aligned}
$$

with the radiative rate coefficient $\beta_{\mathrm{ph}}$ (in $\mathrm{s}^{-1}$ ) through the CBR, defined by:

$\beta_{\mathrm{ph}}=\frac{8 \pi}{c^{2}} \int_{v_{\mathrm{th}}}^{\infty} \sigma_{\mathrm{ph}}(v) \frac{v^{2} \mathrm{~d} v}{\exp \left(h v / k T_{\mathrm{r}}\right)-1}$,

where $v_{\text {th }}$ is the threshold energy for which photoionization is possible (here $h v_{\text {th }}=13.6 \mathrm{eV}$ ). We take the frequency dependent cross section $\sigma_{\mathrm{ph}}$ (in $\mathrm{cm}^{2}$ ) given by Abel et al. (1997):

$$
\begin{aligned}
& \sigma_{\text {th }}= 6.30 \times 10^{-18}\left(\frac{v}{v_{\text {th }}}\right)^{4} \frac{\exp [4-4 \arctan (\epsilon) / \epsilon]}{1-\exp (-2 \pi / \epsilon)} \\
& \text { where } \epsilon=\sqrt{\frac{v}{v_{\text {th }}}-1 .} .
\end{aligned}
$$




\section{References}

Abel, T., Anninos, P., Zhang, Y., \& Norman, M. 1997, New Astron., 2,181

Abel, T., Bryan, G., \& Norman, M. 2000, ApJ, 540, 39

Allin, J. E., Hare, W. F. J., \& MacDonald, R. E. 1955, Phys. Rev., 98, 554

Bahcall, N., \& Fan, X. 1998, ApJ, 504, 1

Carroll, S., Press, W., \& Turner, E. 1992, ARA\&A, 30, 499

Combes, F., \& Pfenniger, D. 1998, Mem. della Soc. Astron. It., 69, 413

Dalgarno, A., \& Lepp, S. 1987, in Molecular Astrophysics, ed. T. W. Hartquist (Cambridge University Press), 473

De Bernardis, P., Ade, P. A. R., Bock, J. J., et al. 2000, Nature, 404, 955

Flower, D., \& Pineau des Forêts, G. 2000, MNRAS, 316, 901

Freedman, W. 2000, Phys. Rep., 333, 13

Galli, D., \& Palla, F. 1998, A\&A, 335, 403

Galli, D., \& Palla, F. 2002 [astro-ph/0202329]

Gear, W. 1971, in Numerical initial value problems in ordinary differential equations (ed. Prentice-Hall)

Hegyi, \& D., Olive, K. 1986, ApJ, 303, 56

Hirasawa, T., Aizu, K., \& Taketani, M. 1969, Prog. Theor. Phys., 41, 835

Hollenbach, D., \& Salpeter, E. 1970, J. Chem. Phys., 53, 79

Hollenbach, D., \& Salpeter, E. 1971, ApJ, 163, 155

Hoyle, F., Wickramasinghe, N., \& Reddish, V. 1968, Nature, 218, 1124

Jaffe, A., Ade, P. A., Bock, J. J., et al. 2001, Phys. Rev. Lett., 86, 3475

Lahav, O. 1986, MNRAS, 220, 259

L'Air Liquide 1976, Gas Encyclopedia (ed. Elsevier)

Latter, W., \& Black, J. 1991, ApJ, 371, 161

Lee, S. Y., Lee, S., \& Gaines, J. R. 1988, Phys. Rev. B, 37, 2357

Lee, T., Gowland, L., \& Reddish, V. 1971, Nature Phys. Sci., 231, 193

Lepp, S., \& Shull, M. 1984, ApJ, 280, 465

Lepp, S., Stancil, P., \& Dalgarno, A. 2002, J. Phys. B, 35, R57

Longair, M. 1995, in The Deep Universe, Saas-Fee Advanced Course 23, ed. B. Binggeli, \& R. Buser (Springer Verlag), 317

Mather, J. C., Cheng, E. S., Cottingham, D. A., et al. 1994, ApJ, 420, 439
Matsuda, T., Sato, H., \& Takeda, H. 1969, Prog. Theor. Phys., 41, 219

Partridge, R. 1995, in 3K: The Cosmic Microwave Background Radiation (Cambridge University Press)

Peebles, P. J. E. 1968, ApJ, 153, 1

Peebles, P. J. E. 2002 [astro-ph/0207347]

Perlmutter, S., Aldering, G., Goldhaber, G., et al. 1999, ApJ, 517, 565

Pfenniger, D., \& Combes, F. 1994, A\&A, 285, 94

Puy, D., Alecian, G., Le Bourlot, J., Léorat, \& J., Pineau des Forêts, G. 1993, A\&A, 267, 337

Puy, D., \& Signore, M. 1996, A\&A, 305, 371

Puy, D., \& Signore, M. 1997, New Astron., 2, 299

Razoumov, A. O., Norman, M. L., Abel, T., \& Scott, D. 2002, ApJ, 572,695

Riess, A., Filippenko, A. V., Challis, P., et al. 1998, AJ, 116, 1009

Sanford S., \& Allamandola, L. 1993, ApJ, 409, L65

Shapiro, P. 1992, in Astrochemistry of Cosmic Phenomena, ed. P. Singh (Kluwer Academic)

Silvera, I. F. 1980, The solid molecular hydrogens in the condensed phase: fundamentals and static properties, Rev. Mod. Phys., 52, 393

Srianand, R., Petitjean, P., \& Ledoux, C. 2000, Nature, 408, 931

Stancil, P., Lepp, S., \& Dalgarno, A. 1996, ApJ, 458, 401

Stancil, P., Lepp, S., \& Dalgarno, A. 1998, ApJ, 509, 1

Takeda, H., Sato, H., \& Matsuda, T. 1969, Prog. Theor. Phys., 41, 840

Tytler, D., O’Meara, J., \& Lubin, D. 2000, Phys. Scr., 85, 12

Uehara, H., Susa, H., Nishi, R., Yamada, M., \& Nakamura, T. 1996, ApJ, 483, L95

Uehara, H., \& Inutsuka, S. 2000, ApJ, 531, L91

Urey, H. 1935, Some thermodynamic properties of hydrogen and deuterium, in Le Prix Nobel en 1934, Stockholm: Kungl. Baklryckenet, 1

van de Hulst, H. C. 1949, Recherche Astron. Utrecht, 11, 1

Varghese, G., Prasad, R. D. G., \& Paddi Reddy, S. 1987, Phys. Rev. A, 35,701

Wardle, M., \& Walker, M. 1999, ApJ, 527, L109

Wetterich, C. 1988, Nucl. Phys. B, 302, 321

Wickramasinghe, N., \& Reddish, V. 1968, Nature, 218, 661

Zwicky, F. 1959, PASP, 71, 468 